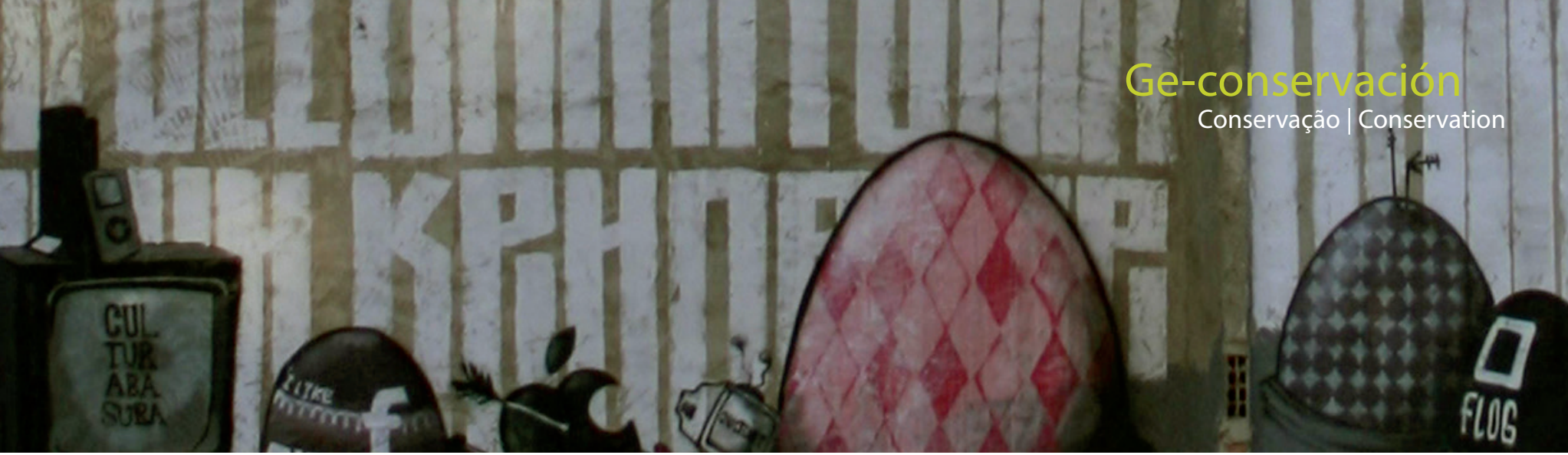

\title{
Etapas del Arte Urbano. Aportaciones para un Protocolo de conservación
}

\author{
Elena García Gayo
}

\begin{abstract}
Resumen: Los protocolos de conservación garantizan el adecuado tratamiento de los bienes culturales. Así como un reglamento de conservación preventiva puede sistematizar acciones que garanticen la correcta exhibición o manipulación de obras en un museo, un protocolo para la conservación de Arte Urbano adaptado a sus características y particularidades, ayudaría a deducir las posibilidades para conservar una obra desde la documentación técnica previa. La detección de los puntos claves que dan valor material a la obra, entre ellos, las condiciones espaciales que le son inherentes y el significado que adquieren para una comunidad de personas, daría pie a una base argumental que justificaría la decisión de conservarla. Para conseguir este objetivo es necesario describir unas etapas de Arte Urbano que faciliten la catalogación de obras.
\end{abstract}

Palabras clave: street art, expresión independiente, arte urbano, murales contemporáneos, protocolo, proto street art, intermural art, conservación

\section{Street art stages, contributions for a preserving protocol}

Abstract: The protocols guarantee the proper treatment of the cultural goods. As a preventive rules may systematize the actions so as to guarantee the suitable exhibition or of masterpieces in a museum, a particular protocol for the maintenance of Street Art, specifically adapted to its personal features and characteristics, would certainly provide with the possibilities to preserve a work of art from its very and previous technical literature. The early detection of the key points that substantiate its material value, among them, the spatial conditions inherent to those as well as the acquaired meaning to a community of individuals would provide with an argumental foundation that would justify the preservation choice. Nonetheless, it is almost mandatory to outline the stages of Street Art to facilitate the categorization of the pieces in particular.

Key words: street art, independent expressions, street/urban art, contemporary murals, protocol, proto street, intramural art, conservation. contemporary interventions.

\section{Introducción}

En los últimos años se tiende a usar el término"arqueología" para definir la recuperación de la memoria de obras desaparecidas y aunque es un término muy visual no es correcto; si la recuperación de piezas arqueológicas abre un camino al conocimiento de civilizaciones pasadas a partir de los artefactos que producen, en el caso del Arte Urbano es más coherente recuperar la memoria mural sentimental, de una generación, con los hechos que provocaron su existencia, el contexto en el que sucedieron y su relación con el entorno o, más concretamente, la de la memoria de un barrio en un punto concreto de su historia. Si se insiste en utilizar el término, lo más correcto sería hablar de "arqueología de la arquitectura" y más concretamente dentro de ella la disciplina encargada, entre otras cosas, de la "lectura de muros y paramentos".

Las acciones que tienen por objeto preservar la memoria están siendo llevadas a cabo por la facilidad de adquirir y divulgar imágenes digitales, pero la conservación de la materia reclama la creación de pautas preventivas que puedan conseguir un proceso de degradación muy lento, hasta la pérdida total. La naturaleza inestable, 
degradada y envejecida, así como las técnicas de ejecución rápidas utilizadas en el Arte Urbano, produce obras que se mantienen un periodo de tiempo demasiado corto; alargarlo, o no, dependerá del examen de sus particularidades. El análisis de sus contradicciones, los aspectos jurídicos o sociales que las protegen, la importancia o levedad de su resistencia material, la motivación que le dio origen y la importancia del contexto, son aspectos que ayudarán a clasificarlas dentro de una tendencia artística que tras cuarenta años de existencia ya no tiene nada de efímera.

\section{Objetivos y metodología}

Describir los parámetros que sirvan como referencia para ayudar al estudio y clasificación de obras de Arte Urbano a través de referencias; esto debe esclarecer si debe ser estudiado desde la Historia del Arte o desde la historia de movimientos urbanos contraculturales; si es susceptible de serlo desde el punto de vista de la conservación de obras efímeras, y si, además, estas herramientas pueden facilitar el análisis pormenorizado de cada caso en particular.

La metodología aplicada se basa en el análisis de entrevistas a artistas cuya ficha técnica fue publicada en la 12a Jornada de Conservación de Arte Contemporáneo del Grupo Español del IIC y que empezó a ser utilizada en 2011 en el V Asalto de Zaragoza, ampliada con entrevistas también a expertos en Arte Urbano desde 2014 (a partir de la base de datos del Grupo Español de Trabajo sobre Conservación y Restauración de Arte Contemporáneo GETCRAC que se puso en marcha en la Diputación Foral de Álava en 1988).

Se ha recurrido al examen de entrevistas publicadas en periódicos digitales y, además, los artículos del grupo de trabajo del GEIIC durante el año y medio de existencia. (Ver gráficos, encuestas y Código Deontológico propuesto por el grupo en el Anexo) y los artículos publicados por los miembros del grupo por el Observatorio de Arte Urbano en la revista digital especializada Mural Street Art Conservation $\mathrm{n}^{\text {os }} 1,2$ y 3. (2015-2016).

\section{Paradojas y paradigmas del Arte Urbano}

Las intervenciones urbanas no se entienden de la misma manera a lo largo de la historia y varía su percepción según desde la disciplina que se aborde. Por ejemplo, desde la arquitectura; según C. Sitte (1889) el arte sirve al embellecimiento de las ciudades, y siguiendo los preceptos de R. Unwin el arte urbano debe ser la expresión de la vida de la comunidad. Con sus choques conceptuales, ambos autores lo definen desde el punto de vista de arquitectos urbanistas que cuentan con la síntesis de todas las áreas del diseño: la arquitectura, la jardinería, la pintura, entre otras, expresan la percepción intelectual de las intervenciones en la calle y cuáles deben ser sus reglas.
Si se tiene en cuenta la evolución de criterios de las disciplinas involucradas y que las obras son objetos jurídicos que forman parte de estructuras urbanas complejas ordenadas, se crean una serie de paradojas y paradigmas a su alrededor que hay que describir para poder examinar.

Entre las múltiples acepciones que definen las intervenciones artísticas producidas en la calle, la más controvertida es la de ilegalidad que, unida al valor artístico que la identifica como arte actual, constituyen la posible contradicción que enfrenta y anima la relación de los artistas con el público. “¿No será el arte verdadero una cosa clandestina?" se preguntaba su autor al analizar el movimiento internacional del Graffiti (Ramírez, J.A: 1992: 206).

De esta manera, el Arte Urbano se ha ido instalando en la sociedad que lo absorbe y transforma. Esta cercanía hace que desde las intervenciones ilegales hasta las más sociales el público se convierta en principio y fin de las propuestas y en dos agentes condenados a entenderse porque están emplazados a un futuro diálogo artístico por la conservación.

Por otra parte, si el arte debe tener un valor económico, que es la eterna cruz de todo lo artístico, al Arte Urbano reconocido como ilegal le viene bien la gratuidad; no se puede ser un alma más pura ni estar mejor condenado, a la indigencia, que cuando se es artista, urbano e ilegal. Pero como el reconocimiento social viene dado por la valoración económica y en los artistas no hay excepciones, se puede estar en la calle y en la galería con la consiguiente ambivalencia: la ilegalidad da respeto y mantiene la vigencia dentro del colectivo, mientras que la legalidad facilita su pervivencia y el reconocimiento social. Las obras de la calle transforman la regla stop, think, go, do en "piensa, crea, actúa y olvida", mientras que las obras de galería se rigen, como es habitual, por el mercado del arte que intentará crear marca comercial de cada artista y aprovechará la fama que le viene dada de la parte urbana. Así, aunque exponer en la calle y en una galería sean cosas diferentes, y a pesar de que los artistas alimenten esta bipolaridad, se trata de dos ámbitos de expresión con posibilidades diferentes. Uniendo esa información es cuando se debe valorar una carrera artística en la que no se podría entender una trayectoria sin la otra.

No todas las variables que interactúan en una performance urbana pueden ser registradas y conservadas. "Una obra de arte urbano no es pintura ni dibujo: es, ante todo, trabajo con un contexto. El material es la propia ciudad. El resultado tiene que ver con el dónde, el cómo y el cuándo, tanto o más que con el qué" (Abarca, J. 2016:4). Aun así, no se cree arriesgado afirmar que casi todos los estudiosos del Graffiti y el Arte Urbano conservarían algunas obras, si hubiera garantías. "Aunque la única forma de conservación del Graffiti que conozco es la fotografía, echo de menos el que no se haya podido conservar alguno de los vagones 
de metro de Nueva York de los 70". (Entrevista a Martha Cooper. Honolulu. Oahu, Hawaii. Febrero de 2014).

\section{Etapas del Arte Urbano}

Gracias a la era tecnológica estamos viviendo en un espacio digital al que nos hemos incorporado como un complemento más de nuestras posibilidades de observación del mundo, conocemos muchas obras de arte a través de este medio y sabemos exactamente dónde se encuentran aunque no salgamos a la calle. Incluso navegando por la red se puede entrar en el interior de muchos museos y tener la sensación de andar por las calles con google earth. Esta experiencia no sustituye a la realidad de pasear $y$, aunque ayuda a planificar exactamente dónde se quiere ir, evita en gran medida la sorpresa, uno de los fundamentos de esta manifestación. Si la red ha facilitado las posibilidades de difusión de obras de la calle, también ha modificado la percepción del mundo y nuestra participación en él. De hecho, en la actualidad, experimentar el Arte Urbano ha dejado de ser un hallazgo casual para convertirse en la búsqueda de un rastro virtual.

Bajo la mirada del observador, todo aquello que se sale de las fórmulas de diseño de la señalética urbana oficial y está en la calle puede ser considerado Arte Urbano, y es así porque según Beuys: "cada hombre es un artista", pero con una mirada analítica no se podría llamar Arte Urbano a todo sin "establecer un diálogo entre la micro y la macrohistoria, que conecta presente y pasado, prestando atención a precedentes, analogías con otros marcos culturales, lo particular y lo universal, lo heredado y lo legado, y dando luz a aspectos como la influencia cosmovisional, política o económica" (Figueroa 2016:12) para lo cual es necesario crear una metodología que sirva de estructura para su descripción. Tal y como sugiere Rafael Schacter (2016) recientemente si el Arte Urbano es finalmente reconocido como un periodo más del arte, éste, tiene unas etapas dentro de las cuales se pueden describir diferentes tipos de obras que se producen en la calle. Las diferencias no sólo deberían afectar a su tipología sino a su motivación y situación jurídica, porque estos aspectos dan pie a muchas diferencias de concepto y ejecución. Este autor propone el análisis de divisiones tales como "Proto Arte Urbano" o"Arte Intermural" que en el caso de la conservación, ayudaría a determinar diferencias en las producciones ejecutadas en el espacio público desde finales de los años sesenta hasta hoy y aportar claridad, también, para comparar las intervenciones entre sí y catalogar las obras producidas. Así pues, se propone la división en las siguientes etapas: Proto Arte Urbano, Arte Urbano, Muralismo Contemporáneo e Intervenciones Contemporáneas.

-Proto Arte Urbano, quedaría definido por las primeras actuaciones, las pioneras, aquellas que no imaginaban dar lugar a un movimiento internacional como las de Ernest Pignon de 1966, cuando probablemente ya se hacían oír las primeras voces que desencadenaron en las revueltas de Mayo del 68. Las obras del mismo autor, de 1971, cuando publicita y explica su exposición en galería. Aquellas, en las que después relaciona espacios y hechos históricos clave en la historia, situándolos en su espacio original ya descontextualizado por el paso del tiempo, y persigue la conservación de la memoria de hechos históricos sangrientos de Francia. Estas intervenciones, constituyen un tipo de expresión comprometida, como la anti celebración de los "contra-monumentos" que fue una propuesta expositiva producida por Hans Haacke.

Se pueden considerar así las primeras obras de Blek Le Rat o las acciones relacionadas con La Internacional Situacionista, que tienen que ver con la exploración del territorio y en las que hoy en día Eltono desarrolla algunas de sus experiencias urbanas, o las de Matthias Wermke y Misha Leinkauf en las líneas de metro fuera de servicio en Alemania y las de Les Frères Ripoulain en Francia.

Las incursiones de artistas muy conocidos en el Arte Urbano ofrecen claridad sobre cómo se produjeron las primeras intervenciones en la calle a partir de los años ochenta. Además de Keith Haring o Michel Basquiat, las sombras inquietantes de Richard Hamilton en el East Village de Nueva York aparecen en 1982 y las de Dennis Adams, que actuó a mediados de los ochenta en las paradas de autobús con un discurso artivista (contracción gramatical de arte y activismo).

Todos ellos intentaban romper la monotonía de las calles, esconderse para ser encontrados y con ello provocar al aburrido transeúnte saturado de información. La esencia que une a todas estas actuaciones, lo que se desprende de ellas, es que los artistas buscaban una manera personal de relacionarse con el entorno y son esas particularidades espacio-temporales en las que se producen obras artísticas únicas y de existencia efímera e irrepetible.

-Arte Urbano es el término con el que no queda más remedio que identificar, como referencia, las obras de Banksy y su entorno porque, se quiera o no, guste más o menos, es el artista que ha hecho posible el salto mediático de esta tendencia a nivel mundial y ha conseguido que se hable del arte de la calle en todos los foros posibles. Su aparición marca un punto de inflexión y ruptura con la influencia situacionista, para entrar en el mundo del espectáculo, en las salas de subastas, forzando su reconocimiento como artista contemporáneo y ser aceptado como artista conceptual. Banksy y Shepard Fairey, encabezan una lista de artistas que van de la calle a la galería y sus obras de la calle a las casas de subasta; con ellos se inicia un cambio en el concepto de intervención urbana independiente y aunque conservan algunas características que los identifican con el Proto Arte Urbano (como la ejecución en lugares de paso y el uso del pequeño y mediano formato, el juego por negar la autoría de algunas de 
sus obras y confirmar otras, a través de una oficina de autentificación de obras como hace Banksy con la web Pest Control) también les relaciona con una pérdida de criterio en favor de las ganancias obtenidas por la comercialización de sus obra.

-Muralismo Contemporáneo es la etapa que servirá para diferenciar las obras que se producen de forma legal, que surgen de la necesidad de expresión en el medio urbano y se convierten en novedad por la apropiación de las instituciones, las marcas y un sin fin de causas sociales. Son obras subvencionadas, en las que la organización actúa ejerciendo el comisariado y aparece una tímida pero clara intención de hacer exposiciones públicas y colección. En estos casos, normalmente no se interfiere en la libertad del artista, y las obras, además de ser encargos, pasan por una selección previa en la que se eligen los participantes o, a la inversa, se aceptan sus propuestas. Esta etapa, en España, puede tener una de sus muchas referencias en las primeras obras murales de un grupo de artistas canarios entre los que se encontraba el artista Manuel Padorno, [Figura 1] ejecutadas en el Paseo de Las Canteras en 1994, cuyas obras estaban basadas en su obra literaria y fueron un reclamo para atraer turismo a la isla. En ese año coincide la creación de la empresa de botes de pintura Montana Colors y posteriormente las convocatorias e intervenciones del Asalto de Zaragoza, Open Walls de Barcelona y una larga lista de festivales y convocatorias públicas y privadas.
Muralismo Contemporáneo o Intervenciones Contemporáneas, ambos son términos para definir una misma época, porque no todas las obras que se generan en la calle son murales, pero se le dé el nombre que se le dé al arte que tiene la calle como contexto, es imprescindible aclarar su situación jurídica y social, porque ambas van a influir en las posibilidades de conservación, como veremos.

\section{De lo jurídico y lo social}

Si algo es constante entre los siglos XX y XXI es la búsqueda de nuevos espacios para la expresión artística y ese es el motivo por el que cada vez más artistas salen de los museos y buscan nuevas formas y fórmulas de exposición. La calle es el ámbito social donde existe una clara necesidad por significar espacios que son cada vez más impersonales y parece el entorno idóneo para crear obstáculos visuales con propuestas artísticas atractivas ${ }^{2}$. El Arte Urbano es un medio útil para vincular socialmente a las personas con el territorio, porque lo interiorizan y"se hacen a sí mismas mediante las propias acciones en un contexto sociocultural e histórico" (Vidal Ty Pol U, 2005:283).

Las actuaciones artísticas murales son susceptibles de tener dos apariencias jurídicas diferentes (ilegal y legal) que son las que deciden su titularidad y protección en el código civil español (Art. 353) ${ }^{3}$ donde se regula el derecho de accesión. Así pues, la propiedad de los bienes

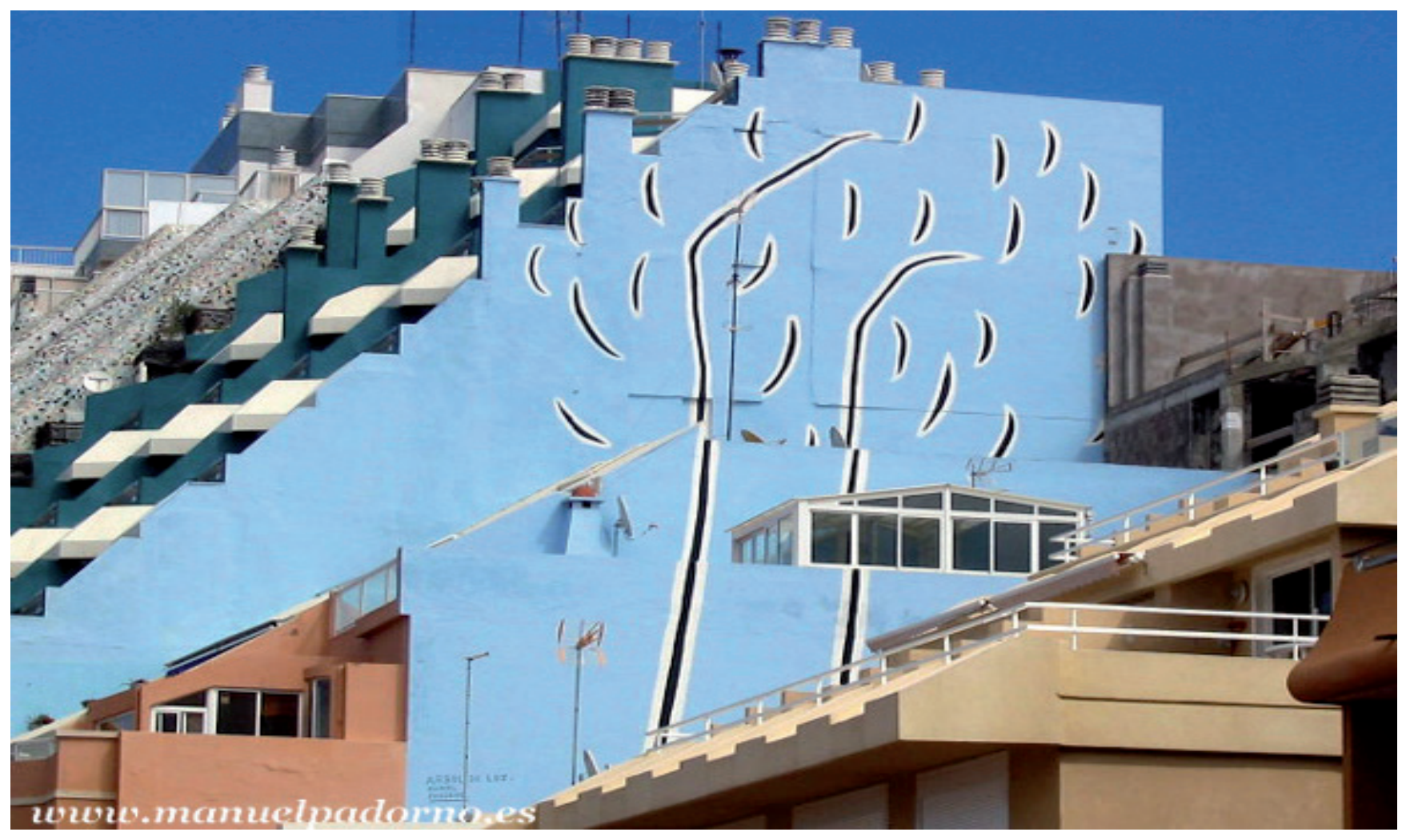

Figura 1. Mural de Manuel Padorno en Las Palmas de Gran Canaria. Árbol de luz. Anamorfosis, 1994. Foto de Patricia Padorno Asociación Cultural Manuel Padorno http://www.manuelpadorno.es/ (15-10-2016). 
da derecho por accesión a todo lo que ellos producen, o se les une o incorpora, natural o artificialmente; es decir, que el propietario del soporte, el muro, puede hacer suya cualquier producción artística que se realice en él. Como contrapartida, por el "derecho moral" mencionado en la Ley de Propiedad Intelectual ${ }^{4}$ por el que un artista puede elegir la forma de divulgación de su obra (art. 14.1) se crea una ambivalencia, por un lado el muro, como soporte, y por otro la capa pictórica, la obra. Estas dos individualidades constituyen una "dimensión dinámica que bajo determinadas circunstancias, cualquiera de las dos, soporte y obra pictórica, es capaz de atraer hacia su órbita la titularidad del objeto". (Sánchez Aristi, R.:2015:33).

Siempre que se habla de la ausencia de derechos de una obra de Arte Urbano se expresa un menosprecio de su valor en favor del valor inmobiliario del muro. Esto, en la obra de Banksy, que es un ejemplo muy conocido, es al revés porque la obra revaloriza, al alza, el valor de mercado del inmueble, tal y como hemos visto en múltiples ejemplo, uno de los últimos el del mural de los espías, The spy booh .

Las obras ilegales son independientes y espontáneas, normalmente de pequeño tamaño, ejecución rápida y una localización cercana a la línea de transeúntes donde, por un lado, son más vulnerables y muy efímeras y por otro, invitan a la participación, opinión o expresión sobre ellas.

Además de estos dos tipos jurídicos mencionados, se habla de obras alegales aunque llamarlas así es una licencia poética, pues se refiere a las que están en espacios muy degradados y pasan desapercibidas; su titular no llega nunca a ejercer el derecho de reclamación y muchas veces el propietario es el Estado, ya que la ley no deja vacía esta posibilidad de titularidad de bienes inmuebles. Son espacios en los que se generan obras ilegales pero no existe un conflicto de intereses al estar ejecutadas en muros abandonados, edificios esperando ser demolidos, fábricas sin actividad o muros provisionales.

Por el contrario, aquellas intervenciones realizadas en soportes muebles y abandonadas sí que son susceptibles de ser adquiridas por "ocupación" y conseguir así el derecho de exposición pública, según el artículo 56.2 de la Ley de Propiedad Intelectual.

Aparte de estas acepciones jurídicas se debe contar con la intervención del público, que tiene derecho de opinión y opina que no todas las propuestas son bien recibidas. Tal y como planteó Laura Luque Rodrigo con su intervención ¿El público decide? dentro de la convocatoria Vincularte. Encuentro abierto sobre Arte Urbano, organizado por el grupo de trabajo del GE-IIC y celebrado en La Casa Encendida de Madrid en Marzo de 2016. (Ver resumen de las encuestas realizadas por el grupo en el ANEXO). Eso no significa que una obra no deba llevarse a cabo; pero el artista va a estar siempre en desventaja de cara a la conservación si el tema elegido no gusta, por mostrar aspectos menos amables del ser humano o simplemente porque no entra dentro de unos cánones estéticos particulares. Cuando lo que se quiere expresar en un muro (que es el punto de vista del artista) y lo que se quiere ver a diario pintado en él (que es el punto de vista de la gente que vive frente a esa pared) son intereses enfrentados, la decisión que tiene respaldo legal es la decisión del propietario del muro. [Figura 2]

El arte no siempre es agradable o decorativo y la libertad de no ser agredido en las vistas cotidianas depende de la sensibilidad, es una causa subjetiva. Esta es una de las paradojas insalvables y el problema no es baladí porque hace que las representaciones no puedan ser todo lo independientes que debieran. Si la acción publicitaria no se puede evitar y no existe defensa legal que respalde el derecho a las propias vistas desde una vivienda, consecuentemente tampoco estará legislada la temática en un mural artístico.

La diferencia entre estos dos casos es que los murales sí que son eliminados mientras que las vallas de propaganda
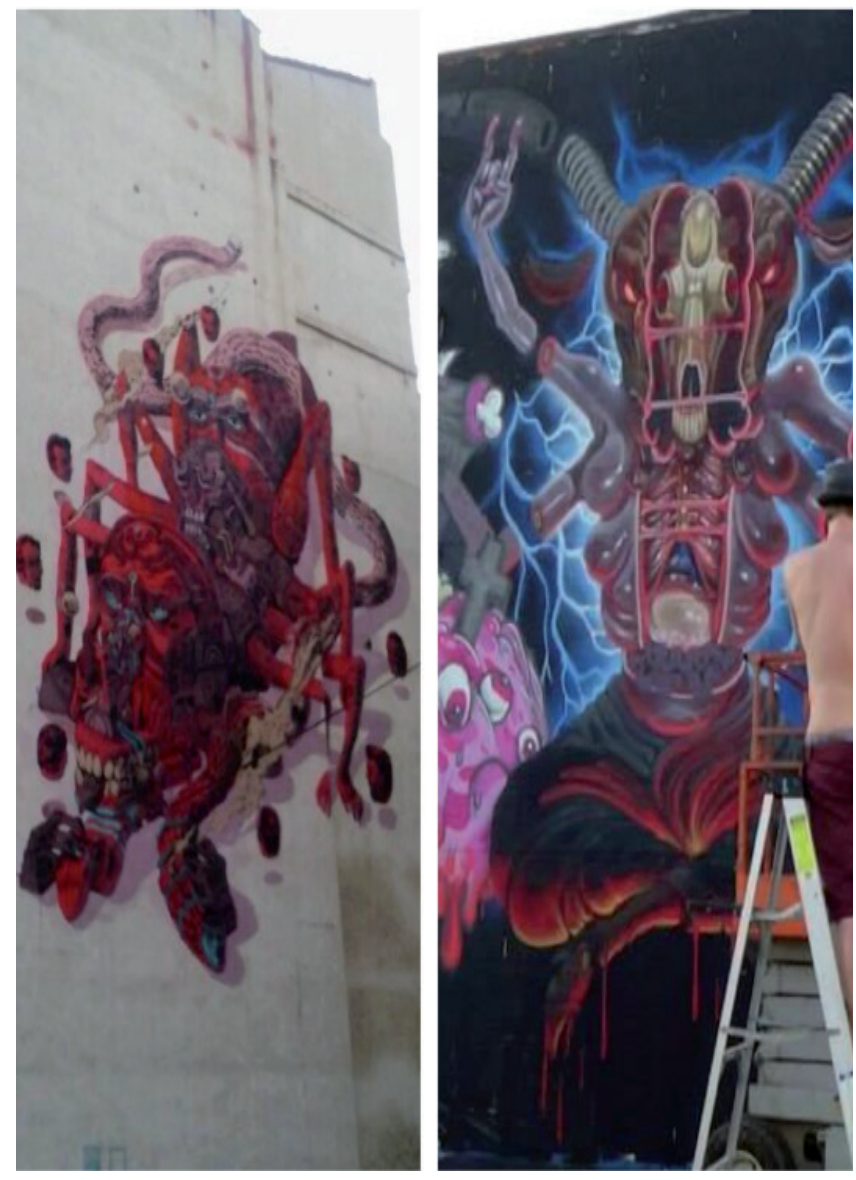

Figura 2. Obras borradas por decisión de los vecinos o el dueño del muro. Izquierda, de Smithe en el Asalto de Zaragoza y derecha, de Nychos en Pow! Wow! Hawaii. Honolulu. Fotos de la autora. 
no, al haber adquirido unos derechos comerciales. Quizá se debería hablar, también, de las razones del que ostenta el poder, decisivas, como bien se ha visto a lo largo de la historia. (Brihuega, J. 1996: 109-126).

En el hipotético caso de que las Intervenciones Contemporáneas sean a la carta, si se ponen límites a la expresión artística, la calle se convertirá en una mera piel amable y decorativa, invisible y superflua de la arquitectura del siglo XXI, al servicio de intereses espurios, a la que por economía de materiales se pedirá cada vez más durabilidad, resistencia y neutralidad, sobre todo neutralidad, para poder soportar el paso de los años y los gustos amables de la sociedad. [Figura 3]

Esa piel efímera, marcada por el ritmo vertiginoso de los acontecimientos, se resiste en muchas ocasiones a ser neutra y salta a la vista aún por encima de la arquitectura, a veces anodina, y reivindica contra todo pronóstico la posibilidad de trascendencia material. Las obras que lo consiguen se convierten en hitos generacionales y documentos de extraordinario valor.

Como ya es sobradamente conocido el caso de la documentadísima obra "Todos juntos podemos parar el sida" de Keith Haring en el histórico barrio del Raval de Barcelona (1989) fué una obra autogestionada pero realizada con permiso del Ayuntamiento de Barcelona y cuya memoria se decidió preservar haciendo, en 1992, un calco que se guarda en el Museo de Arte Contemporáneo de Barcelona (MACBA). La obra tenía un importante trasfondo social, el sida azotaba a la sociedad y este barrio estaba muy degradado. El edificio se tiró, la obra dejó de existir pero el calco garantiza que puede ser reproducida y se ha convertido en un objeto musealizado como registro de la memoria colectiva de una generación [Figura 4]

Todo este recorrido histórico quiere ser un ejemplo de cómo una protección afectará siempre a todos los aspectos contenidos en una obra artística. En la de Haring los derechos de reproducción deben ser de la Fundación Keith Haring que custodia su legado, puesto que se menciona en la reproducción de 2014, coincidiendo con el 25 aniversario de la realización del mural. Es la tercera vez que se reproduce.

Durante la ejecución (en 1989) Haring llevaba un cuaderno de anotaciones en el que describe lo que podría considerarse como una performance que merece la pena mencionarse: "He tardado cinco horas en pintarlo, como había previsto. La pared tenía una inclinación extraña en la que era difícil pintar, pero una de las cosas que más me gustan de este trabajo es la adaptabilidad (física) que requiere. He encontrado una postura que me permitía pintar de una forma homogénea y equilibrada. Algunas de las mejores fotos de este mural reflejan el lenguaje del cuerpo y las posturas que adopto para pintar.» (Web del MACBA).

En este caso, si el contenido social no fuera tan importante, cabría hacerse una pregunta: ¿podría existir infidelidad con el original en la simplificación de un calco que puede ser

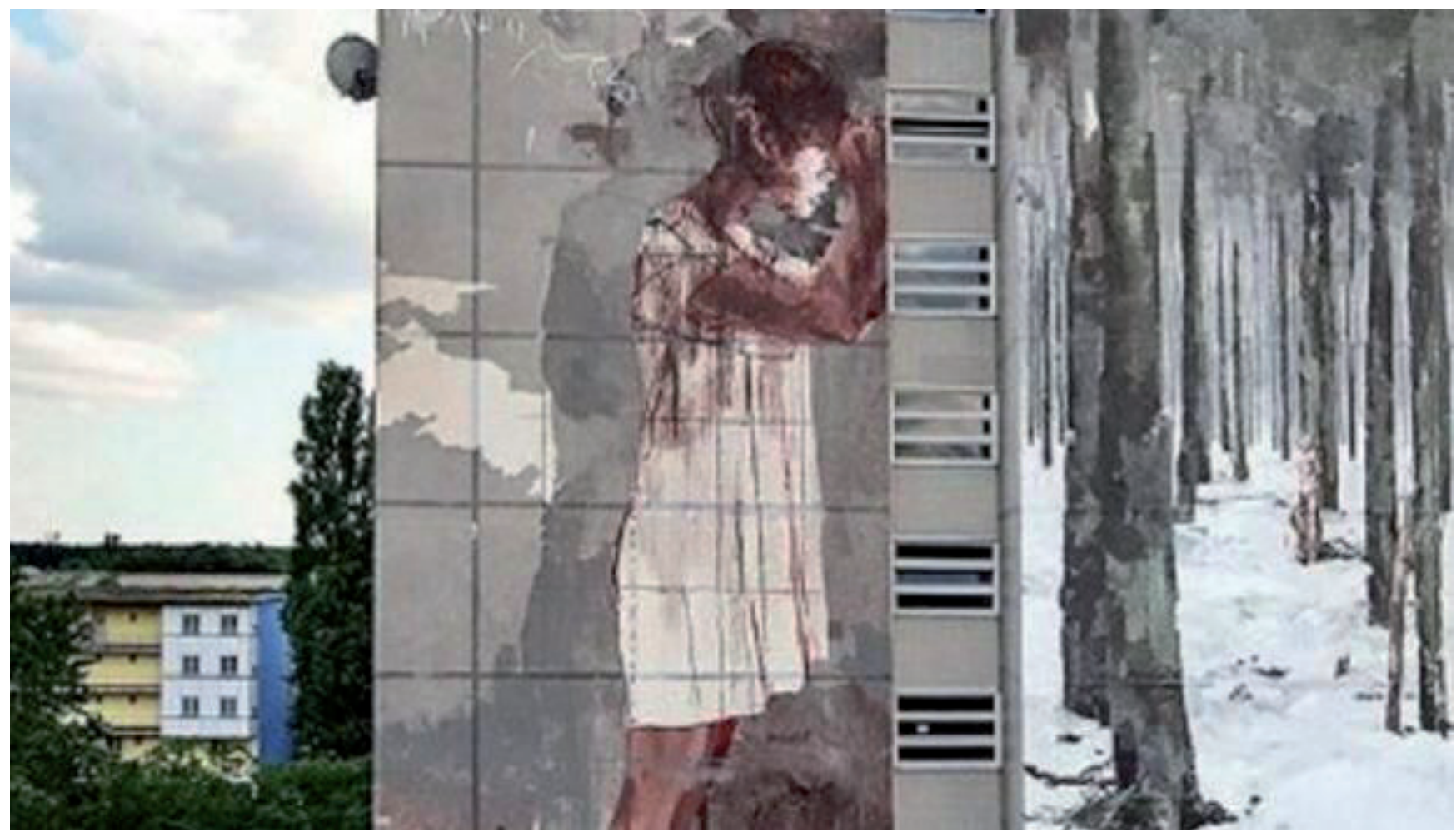

Figura 3. Gonzalo Borondo. Un mural de un artista español causa polémica en Berlín. ABC. 22-06-2016. Foto EFE. ABC Cultura https://goo. gl/MEhacB (15-10-2016). 


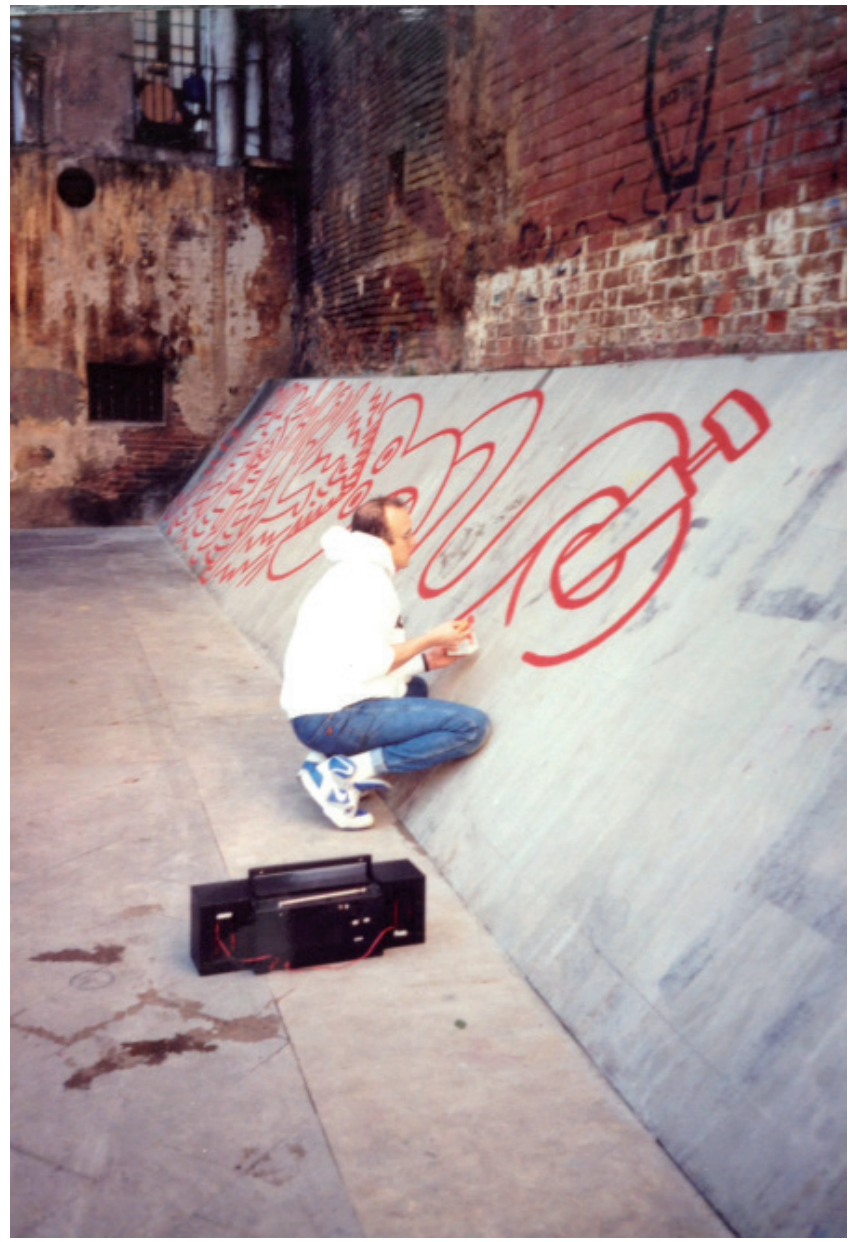

Figura 4. Keith Haring haciendo el mural 'Todos juntos podemos parar el sida', 27 de febrero de 1989, Barcelona. Foto: Montse Guillem. Viene de la página web del MACBA https://goo.gl/ I5PNkA.

reproducido en lugares diferentes y tantas veces como se quiera? Por el contrario, teniendo en cuenta la repercusión del hecho, la enorme difusión mediática de la idea ¿no es el propio acto de reproducción parte importante de la obra?

\section{Obsolescencia programada y levedad de lo efímero}

El espacio público es un palimpsesto en el que las distintas generaciones de artistas van modulando sus intervenciones entre eternos volúmenes arquitectónicos y expresiones plásticas que se complementan e incluso refuerzan; pero al contrario que en la arquitectura, en el soporte de las obras de Arte Urbano se renuncia a la búsqueda de la eternidad, y las producciones parecen tener los días contados desde el mismo momento de su aparición. Conceptualmente y después de dos décadas de existencia artística mediática, sobrevive el juego por vulnerar los límites sociales establecidos y comprobar el grado de represión de cada momento. Lo efímero tiene su sentido porque las obras son abandonadas a su suerte en espacios bien ordenados y neutros, cuya limpieza tiene su ayuda en un plan cultural bien trazado para "crear espacios absolutos para sociedades pluralistas, móviles, agnósticas y desideologizadas"
(Ramírez, J.A.1992:153-172) Quizá esta es la razón de la existencia del Arte Urbano y el Graffiti, que aparecen como respuesta artística a la cultura oficial de los siglos XX y XXI y ha conseguido afianzarse en la memoria colectiva de varias generaciones como una forma de expresión independiente, abierta y no impuesta.

El Arte Urbano es una manifestación cultural actual que no renuncia a formar parte de la historia, pero teme formar parte del eterno dilema de conservación que implicaría introducir cambios permanentes en la evolución urbana; para evitarlo, se hace el harakiri programando la desaparición de sus obras mediante el abandono a su suerte. Antes de morir, muchas de ellas consiguen dejar rastro digital de su esencia sentimental, pero a duras penas se pueden examinar en un monitor las superficies desgastadas, viejas y degradadas o el empaste y naturaleza de la pintura, de unos soportes a los que se ofrece una nueva oportunidad de supervivencia en forma de soporte artístico al que se le atribuye un papel fundamental.

No se puede decir que esta debilidad material facilite la tarea a la hora de decidir un plan de conservación, pero la elección contraria sí que puede llegar a comunicar un deseo de supervivencia. Ahí encuentran su sentido las obras expuestas en la calle y que tienen un acceso complicado en altura, al resguardo de la luz solar directa, la elección de orientación y superficies bien conservadas, ejecutadas con pinturas de calidad; estas obras son rastreadas por los seguidores de tesoros urbanos y sus particularidades pueden ser consideradas como una declaración de intenciones en cuanto a los deseos de conservación. Ningún artista en estas circunstancias negaría haber estudiado esa posibilidad alguna vez.

\section{Motivación, el Big Bang}

Los artistas que se expresan en la calle o en galería no son una unidad indisoluble; muchos no forman parte de ningún colectivo artístico y no hay nexos comunes entre ellos. Aun así, y por una necesidad de simplificación, se ha identificado al artista con el contexto en el que transcurre esta especie de performance urbana, llamándolos a todos artistas urbanos o street artist, cuando la motivación tiene tanto o más peso y, como hemos visto, la forma de usar el espacio público es fundamental. (Vermibus. 2016. Encuentro abierto sobre Arte Urbano. Organizado por el grupo de trabajo del GE-IIC y celebrado en La Casa Encendida de Madrid. Marzo de 2016) Así pues, si la motivación y el proceso creativo son dos piedras angulares para cualquier obra artística en la calle, el contexto tiene un papel decisivo porque añade la posibilidad de improvisación y es imposible generalizar tanto y etiquetar a los artistas por el entorno en el que actúan.

Así pues, si "cualquier actividad realizada sin autorización que, sin embargo, exija su difusión pública debe entenderse primordialmente como una modalidad de discurso" 
(McCormick, C. 2010:16) esto implica el análisis de todo el proceso de la obra incluida su difusión y registrar qué implicación conceptual ha podido tener en el discurso del artista. Según Duchamp, el "coeficiente de arte personal es una relación aritmética entre lo inexpresado pero intentado y lo expresado no intencionadamente" (Duchamp, M: Conferencia en la Federación Americana de Artes en 1957). Esta descripción cobra un especial valor cuando se interactúa en un medio tan rico en propuestas y variables que pueden llegar a influir en el artista a lo largo del proceso de ejecución.

Esta evolución creativa es el primer análisis a realizar sobre una obra artística susceptible de ser conservada, se tenga o no contacto con los artistas, porque siempre hay medios para desencriptar su esencia y conseguir la clave que aporte la información necesaria sobre la razón de su existencia, para situarla en un momento y lugar concretos. El artista o su entorno cercano, el barrio que vivió su aparición y las circunstancias que la envolvieron, pueden ser recogidas de formas diferentes y son imprescindibles para averiguar qué obras son susceptibles de preservarse y cuáles no.

"Los artistas urbanos pueden querer embellecer la ciudad, expresar sentimientos, crear juegos irónicos, lanzar mensajes encriptados o reflexiones críticas" (Prieto, Diana. 2016, pag Web) y se entiende que sus intervenciones deben permanecer el mayor tiempo posible siempre que su contexto no se desvirtúe: caminar por la ciudad e interactuar con el entorno, interpretar el espacio, artivismo, la conquista en tres dimensiones, reivindicar el derecho de expresión, relacionarse, mejorar la vida de barrios socialmente degradados, participar en convocatorias públicas; son algunas de las infinitas posibilidades que pueden llegar a motivar la intervención urbana actual.

\section{Contexto e historia del espacio}

Los artistas participan en muchos proyectos con la conciencia de realizar un trabajo público, donde los murales son la piedra angular que ofrece la visibilidad necesaria, están muy expuestos a la opinión pública y sus obras pueden llegar a ser manipuladas si después cambia el sentido del contexto5."La ubicación lo es todo; el contexto y el contenido son, a fin de cuentas, la diferencia más palpable entre las pintadas en la puerta de un lavabo y (...) el puente de Brooklyn" (McCormick C. 2010:51).

Cuando un barrio muy degradado socialmente es objeto de una reforma para desplazar a la población con menos recursos y convertirlo en un barrio de moda, el primer paso es ofrecer espacios baratos a jóvenes con profesiones liberales y pintar grandes murales. Automáticamente se produce una elitización residencial y un cambio del precio de la vivienda. Aunque los artistas que participan son generadores de ese cambio social cuyo contexto es transformado, hay que tener en cuenta, por un lado, que los artistas se han profesionalizado, ahora son muralistas y las obras son de encargo y, por otro lado, existe un intercambio económico en el que el que paga establece unas condiciones; una de ellas es la cesión de los derechos de imagen y propiedad intelectual de la obra. (Entrevista a Teresa LatuszewskaSyrda gestora de la Fundación Galeria Urban Forms. Łódz. Polonia. Diciembre de 2015).

Sirva como ejemplo los encargos murales del Soho de Málaga; para unos es una oportunidad única de expresión artística al aire libre y para otros un potente aparato al servicio de la gentrificación, tal y como reflexiona Rogelio López Cuenca: “¿Nadie le explicó a Fairey por qué "the art community here in Málaga" supuestamente había decidido poner el huevo precisamente allí? ¿No le hablaron de la transustanciación del "Ensanche" en "Soho"? ¿No le sonaría a otras experiencias de instrumentalización del arte y la cultura como coartada legitimadora de obscenas operaciones de especulación inmobiliaria?" (Web Cuenca Alternativa 2014).

El contexto condiciona el trabajo y la selección de artistas se hace a través de Internet, haciendo un seguimiento de los más populares del momento, no hay que saber demasiado sobre Arte Urbano, porque se trata de encontrar las ofertas más atractivas del mercado internacional. Así es como se llevan a cabo obras de ejecución muy rápida, cuyo encargo no permite que exista demasiada documentación previa por parte de los artistas invitados y así es como los espacios que podrían tener una especial significación local son modificados para crear nuevos contextos. Por primera vez y a través de obras murales de grandes dimensiones se busca que las obras contextualicen de forma atractiva un proyecto futuro, que muchas veces tiene un trasfondo de especulación inmobiliaria.

\section{Conservar es una decisión con implicaciones}

Es evidente que la posibilidad de conservación de Arte Urbano es un planteamiento que debe ir dirigido a unas cuantas obras elegidas y que plantear la conservación o restauración de forma masiva, musealizando la calle, no sería posible ni siquiera aconsejable; pensar esto sólo puede ser considerado una ingenuidad o desconocimiento del proceso de degradación de los materiales que se usan en un medio tan agresivo para ellos, además de una defectuosa valoración del contexto dinámico en el que se desarrolla el Arte Urbano.

La conservación material sólo puede aspirar a alargar la vida a las obras que ya de por sí estén más protegidas de las inclemencias del tiempo. Una vez decidido llevar a cabo un proceso de conservación, es el estudio de los materiales que la constituyen el que va a decidir las posibilidades reales de supervivencia y cómo se puede intervenir para mantenerla durante más tiempo; esto implicaría conseguir una estabilidad que permitiera una degradación muy lenta del color y no por desprendimientos de la materia del soporte, que implicaría una pérdida brusca y por tanto más traumática. 
Tener en cuenta las cuestiones propuestas hace que la catalogación de obras y el análisis de las posibilidades de protección sean complementarios. Así pues, parece lógico pensar que una vez reconocido el Arte Urbano como un período del arte actual algunas obras podrían ver alterada su situación jurídica, ya que el reconocimiento que lleva implícito todo proyecto de conservación las afectaría directamente, al ser aceptadas por los propietarios de los soportes. A partir de ahí se establecería el seguimiento de su degradación dentro de un plan de conservación preventiva.

Quizá no todas las obras en las que se pone el foco puedan soportar sobre sí la idea de este cambio tan radical; por eso, y para no interferir en los derechos morales que se reconocen a todas las obras artísticas, la decisión de alargarles la vida debe contar con el permiso del artista, que debe estar involucrado en el proyecto. Lo contrario, puede dejar a su suerte obras arrancadas de la calle, que son expuestas fuera de contexto, como es el caso de las obras de $B L U$, trasladadas de las calles de Bolonia al museo, aun cuando se ha llevado a cabo con una enorme respuesta popular en contra. ${ }^{6}$

En este caso, tal y como menciona Young, A. (2014:155) "el Arte Urbano que accede a la galería adquiere en su núcleo tres valores culturales: el sentido de la herencia, el carácter igualitario y la rentabilidad". Estos nuevos valores son los que resignificarán la obra y la convertirán en un producto del comisariado, diferente y tutelado.

Por otro lado, la restauración de una obra de Arte Urbano atrae sobre ella la creencia de que puede tener un valor artístico superior al que realmente tiene, porque lo artístico lo es no por razones intrínsecas al objeto, sino porque los usuarios lo reconocen como tal. Lo que tiene interés es que la obra ostenta unos valores de identificación grupal que permite que los individuos se identifiquen con ella" (Formaggio 1976 en Muñoz Viñas, S. 2010:56-57). [Figura 5]

La decisión de conservar depende de los comisarios de exposiciones, la de encargar depende de los gestores de las distintas convocatorias, y borrar, ahora mismo, es competencia municipal; cuyo criterio técnico es el de la "limpieza y eliminación de residuos" dentro del cual las obras se identifican con la suciedad. Este aseo diario de muros se deja a criterio de la empresa y/o la persona que la ejecuta y ni siquiera se utilizan los mismos colores del muro cuando se decide tacharlas, por lo que esa censura no hace más que contribuir a la recreación de un subjetivo arte abstracto que empeora la particular selección. Las normas a seguir se publican en las Ordenanzas Municipales.

En el hipotético caso de la protección de la imagen de obras murales urbanas, la diferencia que se plantea con respecto a otro tipo de obras estaría en la manera en la que debe serlo, cómo y quién las documenta o si se debe compartir públicamente esta documentación que se genera, puesto que son obras de dominio público. ¿Debe

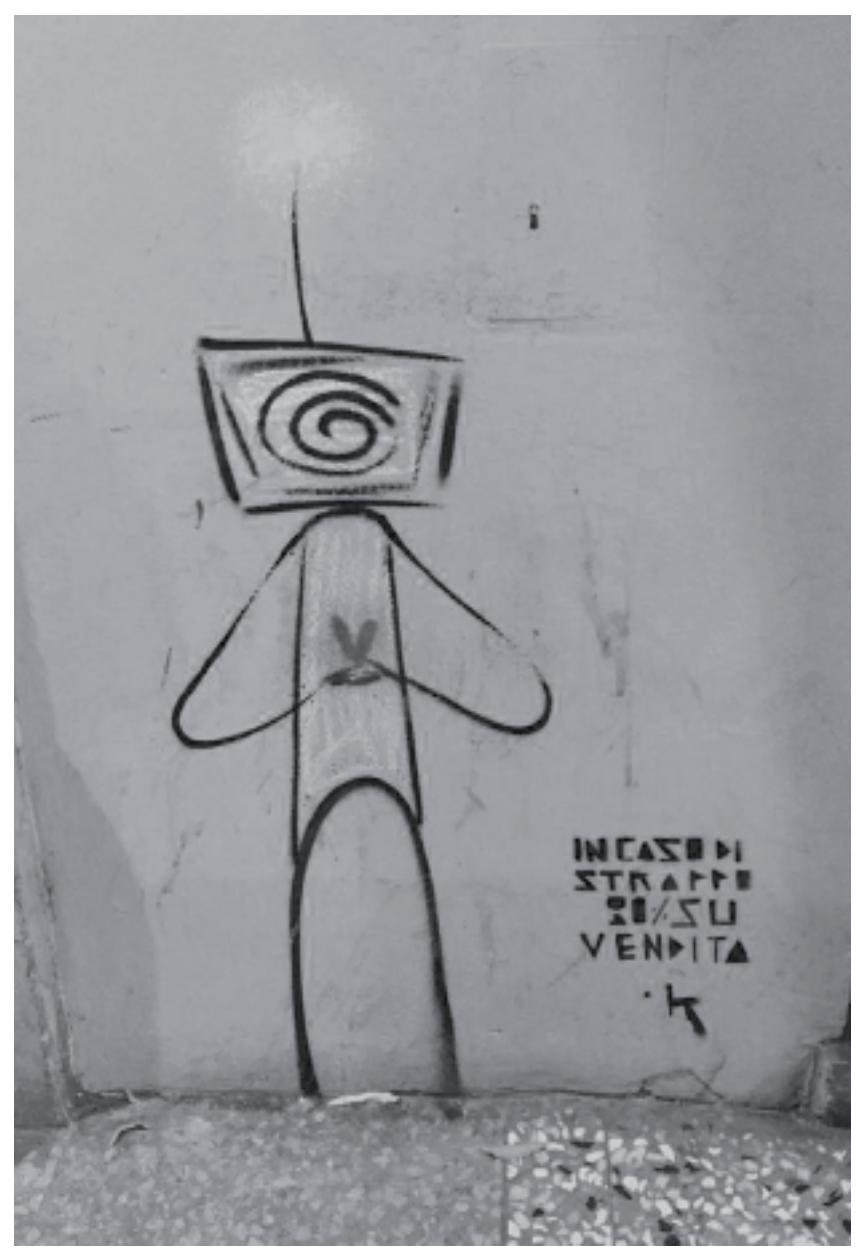

Figura 5. Obra de Exit Enter. Bolonia. "In caso di strappo 80\% su vendita". En referencia al arranque mural de obras de Blu para su exposición en el Palacio Pepoli: Street Art Banksy \& Co. L'arte allo stato urbano. Foto de la autora.

existir un registro municipal de obras que permita la información y seguimiento de las causas de conservación, del proceso de envejecimiento o de las causas que deciden su eliminación?. Llegado a este punto, el mismo trabajo que se realiza en obras de patrimonio histórico artístico sería realizado de cara al público.

Hay algunas consideraciones especiales de los procesos de conservación de Arte Urbano que deben ser tenidas en cuenta; la fundamental es que cualquier obra que haya sido colocada en la calle de forma desinteresada y con la única intención de relacionarse con su entorno, nunca debería ser arrancada para su venta o subasta. No existe ninguna posibilidad de argumentar esta afirmación con respaldo legal, sólo existen los limitados derechos recogidos en la Ley de Propiedad Intelectual, ya mencionados, y un código no escrito de respeto; parece lógico pensar que una obra arrancada de su lugar, que no cuenta con el beneplácito del artista ni de la opinión pública, se va a transformar en un objeto vacío por esas mismas causas. De ahí que el procedimiento a seguir sea tan importante.[Figura 6]

Como norma general, las obras que se han convertido en hitos generacionales y representan a un colectivo o a una 
ciudad, deben ser conservadas in situ el mayor tiempo posible. Para decidir si deben conservarse materialmente y cuáles son los límites en las acciones de preservación deben ser identificadas mediante marcadores objetivos, definiendo una metodología de trabajo a través de la que se genere una completa documentación previa que describa los siguientes aspectos:

- Trayectoria del artista

- La obra en el contexto productivo de su autor

- La relación con la producción de galería

-Motivación y contexto

-El entorno

-Los actores implicados en la conservación (teniendo en cuenta al artista y el propietario del soporte)

- La documentación aportada por el artista y como ha sido recibida la obra en su entorno

- Los intereses en conflicto y valoración de los mismos

-El proyecto de conservación. Valoración de: motivación, contexto y el entorno implicado en la propuesta de conservación

-Opciones de exposición

—La responsabilidad futura sobre los murales. El Comisariado

— La tutela después de la restauración

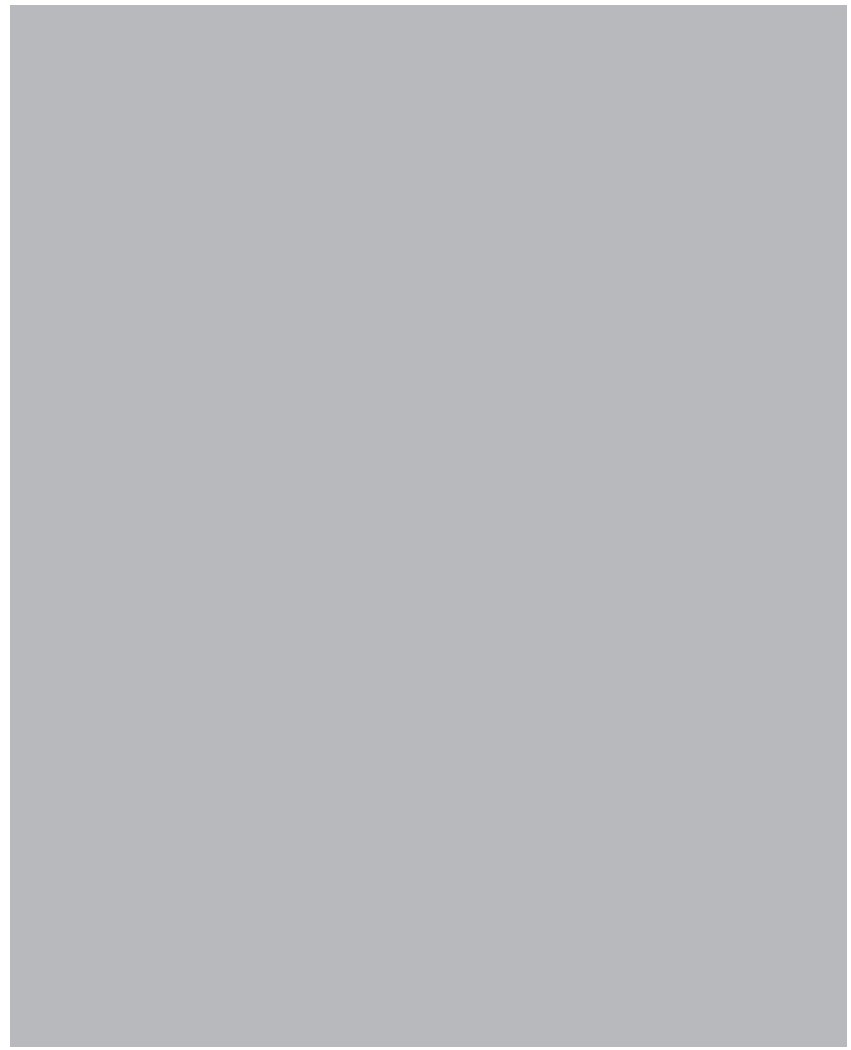

Figura 6. Obra de BLU arrancada de su emplazamiento original para su exposición Street Art Banksy \& Co. L'arte allo stato urbano. Palazzo Pepoli. Bolonia. jun 2016. La foto de la autora ha sido eliminada por voluntad propia ante las dudas de titularidad y para no vulnerar el derecho moral del autor, que no está de acuerdo con el arranque y exposición de su obra en un museo.

\section{Conclusión}

La conservación de Arte Urbano requiere de una ética y planteamientos previos que van a definir y garantizar el estudio en su contexto y la correcta valoración de las posibilidades de conservación.

Por otra parte, y debido a la enorme visibilidad de los grandes murales y las posibilidades de Internet, es necesario considerar que la conservación, aunque sea in situ, puede provocar un desequilibrio entre el valor sentimental y el artístico, por lo tanto, se deben contemplar opciones de difusión que permitan explicar las causas que llevan a su conservación.

Teniendo en cuenta que la decisión de arranque y traslado de una obra mural urbana puede provocar su descontextualización es necesario calibrar si con esta decisión de protección radical se puede llegar a provocar la pérdida de su esencia conceptual. Esta actuación debe estar plenamente justificada por la importancia de la obra en la memoria colectiva de un barrio o una ciudad, del que debe de tener su apoyo, y justificarse a través de una protección en la que el fin didáctico tenga un valor esencial. Por esto, el cambio de estatus jurídico y social, musealización, sólo estaría justificado por unas circunstancias extremas cuyo resultado incluyera su pérdida material inminente.

\section{Referencias:}

[1] La tipografía tiene mucho que ver en las obras de la calle y el título del libro de Heller e llic es muy gráfico de cómo es la actitud para actuar en la calle. Heller, S. Llic', M. Stop, think, go, do. How typography and graphic design influence behavior. Rockport Publisher. 2012

[2] “El arte debe ser libre como libre debe ser el espíritu humano, y recae sobre nosotros, los abogados entrometidos en estas cuestiones, recordar ese principio a nuestros semejantes, a los jueces, a los legisladores y a los formadores de opinión. El derecho es una respuesta, pero también un llamado". Discurso de agradecimiento pronunciado por D. Juan Javier Negri en el acto de entrega en Lisboa, el 7 de junio de 2016 del premio Rodrigo Uría de Derecho del Arte. https://goo.gl/JOeTSQ

[3] R.D. de 24 de julio de 1889, texto de la edición del Código Civil. Ley de 26 de mayo de 2015, vigente hasta el 30 de Junio de 2017.

[4] R.D. 1/1996, de 12 de abril, por el que se aprueba el texto refundido de la Ley de Propiedad Intelectual, regularizando, aclarando y armonizando las disposiciones legales vigentes sobre la materia".

[5] Entrevista a Gonzalo Borondo en la que se ve la manipulación que se hace del trabajo de los muralistas. Synamatix, A. (21-22015) Interview: Borondo on his "animal" exhibition, Street Art and gentrificacion. The Daily Street. "Porque el Consejo ha decidido que esta área debe ser cool y así todo el mundo puede pintar 
en las calles y luego los precios serán altos... ¡Boom! Destruido. Gentrificación."Vamos a poner grandes edificios allí, ahora y todo es más caro. Así que, al final, la gente que empezó a hacer estas cosas para hacer los espacios más públicos para la gente, nos estamos convirtiendo en mercenarios de la gentrificación. Es jodidamente raro, cómo cambian las cosas y cómo el sistema nos utiliza como una herramienta para hacer lo que no queremos". Traducción de la autora. https://goo.gl/Vi6VeD

[6] Entrevistas a los comisarios y restaurador de las obras de BLU arrancadas de la calle para ser expuestas, por Boris. The grifters Journal. Street Art. Banksy \& Co. L'arte allo stato urbano. Palazzo Pepoli. Bolonia Junio 2016. http://thegrifters.org/ inflvencers-interview-christian-omodeo-street-art-banksy-andco-exhibition/ Exposición que ha provocado una respuesta contundente por parte del autor, borrando todos sus murales de la ciudad: Abarca, J. Urbanario: http://urbanario.es/blu-estaborrando-todos-sus-murales-de-bolonia/

\section{Bibliografía}

ABARCA, J. (2016): Conservar o no conservar el arte urbano. Mural Street Art Conservation. Revista especializada digital del Observatorio de Arte Urbano n². (pp12-13) https://issuu.com/ observatoriodearteurbano/docs/mural__2

AZNAR ALMAZÁN, Y.(2009) Martinez Pino, J. Últimas tendencias del arte. Centro de Estudios Ramón Areces. Madrid.

BERTI, G. (2016) Arte efímero para una ciudad con memoria, también efímera...El caso Werens. Mural Street Art Conservation. $\mathrm{n}^{\circ}$ 3. Revista especializada digital. Observatorio de Arte Urbano. Madrid

BEUYS, J. BODENMANN-RITTER, C. Joseph Beuys: cada hombre, un artista: conversaciones en Documenta 5-1972, Editorial Visor, Madrid, 1995.

BRIHUEGA, J. (1996) Arte y Sociedad. Historia de las ideas estéticas y de las teorías estéticas contemporáneas. Vol. II, Madrid, Visor

CLARAMONTE ARRUFAT, J. (2011) Arte de contexto. Nerea

CIANCABILLA, L (2015) The Sight Gallery. Salvaguardia $e$ conservazione della pittura murale urbana contemporanea a Bologna. Bononia University Press.

CIANCABILLA, L. OMODEO, C, (2016) C. Street Art. Banksy \& Co. $L$ 'arte allo stato urbano. Bononia University.

DUCHAMP, M. Conferencia en la Federación Americana de Artes. Duchamp Art News, vol. 56 № 4, 1957. También en: https://goo.gl/ ga1Eec. Universidad de Texas

DUQUE, F. Arte público y espacio político. Arte y estética. Akal, 2001

FIGUEROA SAAVEDRA, F. (2014). El Graffiti de firma. Un recorrido histórico-social por el graffiti de ayer y hoy. Minobitia
FIGUEROA SAAVEDRA, F. (2015) El Historiador del Arte frente al Arte Urbano. Revista especializada digital. Mural Street Art Conservation. Observatorio de Arte Urbano n². (pp12-13) https:// issuu.com/observatoriodearteurbano/docs/mural_2

GARCIA GAYO, E. (2011) ¿Se debe conservar el arte urbano basado en la premisa de: "piensa, crea, actúa y olvida?. Conservación de Arte Contemporáneo. 12a Jornada. Museo Nacional Centro de Arte Reina Sofía y Grupo Español de Conservación. GEIIC.

HELLER, S. LLIC', M. (2012) Stop, think, go, do. How typography and graphic design influence behavior. Rockport Publisher.

LUQUE RODRIGO, L. (2016) ¿El público decide? Revista especializada digital. Mural Street Art Conservation. Observatorio de Arte Urbano n² (pp 32-35) https://issuu.com/observatoriodearteurbano/docs/ mural_3

MCCORMICK, C. (2010) Trespass. Historia del arte urbano no oficial. Taschen

MUÑOZ VIÑAS, S. (2010) Teoría contemporánea de la Restauración. Síntesis.

RAMIREZ, J. A. (1992) Arte y arquitectura en la época del capitalismo triunfante. Editorial Visor. Madrid

RENFREW,C. y BAHN P. 2008. Arqueología. Conceptos clave. Madrid: Ed. Akal S. A.

RUIZ DE ARCAUTE, E. (1993) Bases de datos sobre las técnicas de los artistas contemporáneos y sus criterios de conservación y restauración Boletín Informativo del Instituto Andaluz del Patrimonio Histórico. no 3. Junta de Andalucía. Consejería de Cultura y Medio Ambiente. Dirección General de Bienes Culturales. Sevilla, junio (pags 6 - 7)

SÁNCHEZ ARISTI, R. (2015) La propiedad trenzada: El juego de lo material y lo inmaterial en las obras de arte. Premio Rodrigo de Uría Meruéndano de Derecho del Arte. Anuario Iberoamericano. Cizur Menor, Navarra. Thomson Reuters Aranzadi

SITTE (1889) Construcción de la ciudad según principios artísticos. Fondo antiguo digitalizado. Universidad de Sevilla https://goo. gl/1R1xyR

UNWIN, R. (1909) La práctica del urbanismo. Una introducción al arte de proyectar las ciudades y barrios. G. Gili. Madrid

VVAA.2014. La escena del graffiti en Granada. Pérez Sendra, R (de) Ciengramos. Granada

VIDAL, T y POL URRUTIA,E.(2005) La apropiación del espacio: una propuesta teórica para comprender la vinculación entre las personas y los lugares. Anuario de Psicología, vol. 36, no 3, 281-297. Facultat de Psicologia Universitat de Barcelona. Disponible en https://goo. gl/wfuoaz

WARCLAWEK A.(2008) Graffiti and Street Art. Thames \& Hudson world of art. NY. 
YOUNG, A. (2014) Street art, public city. Law, crime and the urban imagination. Routledge. UK

\section{Vinculos web:}

ABARCA SANCHÍS, J. http://urbanario.es (15-10-2016)

ALDAZ,M. (Coord.). (9-1-2014) Cuenca Alternativa Obey en Málaga. Un análisis de Rogelio López Cuenca (9-1-2014) https://goo.gl/ fHqxjV (15-10-2016)

AYUSO, M. ¿Quienes deciden el valor real de las obras de arte? El Confidencial. 18-2-2012 https://goo.gl/HHscXc (15-10-2016)

BORONDO: http://gonzaloborondo.com/ (15-10-2016)

ELTONO: https://www.eltono.com/es/ (15-10-2016)

GARCIA GAYO, E.. Mural Street Art Conservation. Revista del Observatorio de Arte Urbano. http://observatoriodearteurbano. org/Todos los números disponibles en issuu. (15-10-2016)

GARCIA GAYO, E. Observatorio de Arte Urbano. Ficha técnica y entrevista a artistas (descargable) https://goo.gl/giODsi (15-10-2016)
HIPERALLERGYC. Enttrevista a Rafael Schacter: http://hyperallergic. com/author/rafael-schacter/ (15-10-2016)

MACBA: Haring. Todos juntos podemos parar el sida https://goo.gl/ fGZnne (15-10-2016)

PIGNON ESNST: http://pignon-ernest.com/ (15-10-2016)

PRIETO, DIANA: Preguntas abiertas respuestas cerradas. Input. https://goo.gl/m6UDys (15-10-2016)

MONSERRAT, D. (19-2-2014) Los vecinos obligan a borrar un mural espectacular de la calle Santiago. El periódico de Aragón https:// goo.gl/zyFhSN (15-10-2016)

MONTILLA, C. (13-11-2013) Obey aporta su esperanza a la creación de un Soho malagueño. elmundo https://goo.gl/LSauH6 (15-102016)

PLATIA, N. Athenas, un lienzo en blanco para el arte de la calle. Gounesco. https://goo.gl/leCrqF (15-10-2016)

VERMIBUS http://vermibus.com (15-10-2016)

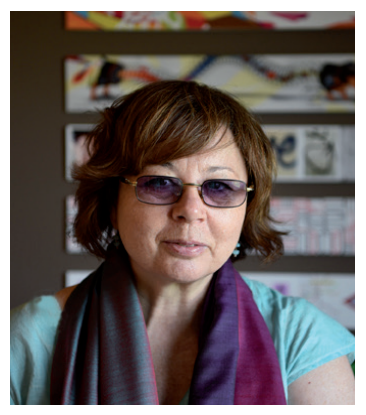

\section{Elena García Gayo}

Conservadora-Restauradora

observatoriodearteurbano@gmail.com

Titulada en Conservación Restauración de Bienes Culturales. ESCRBC de Madrid. Especialidad pintura. 1989. Titular de la plaza de Conservadora-Restauradora en el Servicio de Patrimonio Cultural de la Diputación Provincial de Ciudad Real, dónde desarrolla su labor profesional y de asesoramiento a corporaciones locales en el ámbito de la conservación restauración de patrimonio histórico artístico desde 1990. Paralelamente es coordinadora del grupo de trabajo de Arte Urbano del grupo español del International Institute for Conservation of historic and artistic works, GEIIC. 2015-2016. Asociación internacional sin ánimo de lucro y declarada de utilidad pública.

Principal impulsora de la Plataforma Muelle creada para la conservación de la firma en grosor situada en la c/ Montera de Madrid. Crea y administra el Observatorio de Arte Urbano, con presencia en redes sociales (http://observatoriodearteurbano.org/) y los distintos recursos generados desde el Observatorio: convocatoria participativa Objetivo Muelle, la revista especializada y digital para el análisis de las posibilidades de conservación del Arte Urbano, Mural Street Art Conservation, la cual edita y dirige. Co-Comisariado de la Exposición documental Registros de Arte Urbano 8 en el Centro de Exposiciones de la Diputación de Ciudad Real. 2010. Organiza, con el grupo de Arte Urbano del GE-IIC, el 1er VINCULARTE. Encuentro abierto sobre Arte Urbano, en La Casa Encendida de Madrid. 2016 